\title{
Social and Contextual Areas
}

National Cancer Institute

\section{Source}

National Cancer Institute. Social and Contextual Areas. NCI Thesaurus. Code C15887.

Subcategory of Mind-Body Medicine in CAM classification. This area deals with social, cultural, symbolic and contextual interventions not covered in other areas. For example, the study cross-cultural explanatory models, placebo or "meaning response" research, and caring-based approaches to health care fall into this category. Included are: Placebo, Explanatory models, Caring-based approaches, Community- based approaches, Intuitive diagnosis. 